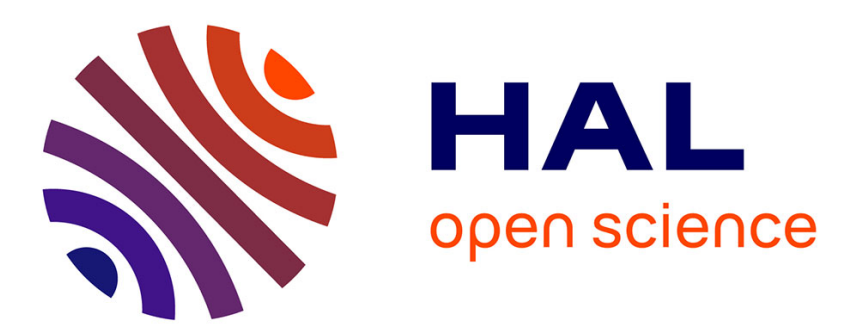

\title{
La notion de tolérance dans les milieux juifs de Metz, d'Isaïe Berr-Bing (1787) au Sermon sur la tolérance de Lazare Wogue (1840)
}

Claire Placial

\section{- To cite this version:}

Claire Placial. La notion de tolérance dans les milieux juifs de Metz, d'Isaïe Berr-Bing (1787) au Sermon sur la tolérance de Lazare Wogue (1840). Etudes Germaniques, 2020, 299 (3), pp.509-519. 10.3917/eger.299.0509 . hal-03508827

\section{HAL Id: hal-03508827 https://hal.science/hal-03508827}

Submitted on 3 Jan 2022

HAL is a multi-disciplinary open access archive for the deposit and dissemination of scientific research documents, whether they are published or not. The documents may come from teaching and research institutions in France or abroad, or from public or private research centers.
L'archive ouverte pluridisciplinaire HAL, est destinée au dépôt et à la diffusion de documents scientifiques de niveau recherche, publiés ou non, émanant des établissements d'enseignement et de recherche français ou étrangers, des laboratoires publics ou privés. 


\section{Claire PLACIAL*}

La notion de tolérance dans les milieux juifs de Metz, d'Isaïe Berr-Bing (1787) au Sermon sur la tolérance de Lazare Wogue (1840)

Quand le Juif de Metz Isaïe Berr-Bing écrit en 1787 la Lettre dans laquelle il préconise la tolérance politique envers les Juifs de France, ce maskil, élève de Mendelssohn s'adresse aux Français chrétiens, et demande l'émancipation des Juifs. Quand, cinquante-trois ans plus tard, le jeune rabbin Lazare Wogue publie son Sermon sur la tolérance, il s'adresse cette fois à ses coreligionnaires messins. Entre-temps, la notion de tolérance a évolué : elle n'a plus le sens politique que lui donnait Berr-Bing, mais intervient dans le contexte des controverses théologiques qui agitent la communauté juive de Lorraine. Wogue utilise ainsi un concept des Lumières française hors de son contexte originel afin de prévenir une scission entre réformistes et conservateurs au sein du judaïsme français.

Wenn der Metzer Jude Isaïe Berr-Bing 1787 die Lettre schreibt, in der er politische Toleranz gegenüber die Juden Frankreichs befürwortet, richtet der Maskil und Schüler Moses Mendelssohns das Wort an die französischen Christen und bittet um die Emanzipation der Juden. Wenn der junge Rabbi Lazare Wogue 53 Jahre später sein Sermon sur la Tolérance (Predigt über die Toleranz) herausgibt, spricht er seine Metzer Glaubensgenossen an. Inzwischen hat sich der Begriff Toleranz verändert: er hat jetzt wenig politischen Sinn, sondern es handelt sich um theologische Streite, die die jüdische Gemeinde Lothringen schütteln. Wogue benutzt also ein Begriff, das aus der französische Aufklärung stammt, außer seines originellen Kontext, um die Spaltung zwischen Konservativer und Reformer innerhalb des französischen Judaismus zu verhindern.

\footnotetext{
*Université de Lorraine, ECRITURES, F-57000 Metz, France ; courriel : claire.placial@univ-lorraine.fr
} 

Le 31 juillet 1841, le jeune Lazare Wogue, encore élève de l'école centrale rabbinique de France alors établie à Metz, futur traducteur du Pentateuque en français, prononce à la Synagogue consistoriale de Metz un «Sermon sur la tolérance » dont l'écho est suffisamment retentissant pour qu'il soit imprimé. Wogue y fait de la tolérance une prescription divine, en redéfinissant les contours d'une notion qui avait pu sembler antithétique avec une conception dogmatique de la religion, mais qui prend une coloration toute particulière dans le contexte de l'émancipation puis de l'assimilation des Juifs de l'Est de la France. C'est à l'évolution des enjeux liés à l'emploi de la notion de " tolérance » dans les milieux juifs messins entre la veille de la Révolution et le milieu du XIX ${ }^{\mathrm{e}}$ siècle que je consacrerai mon propos, en m'attardant sur deux textes en particulier. Le premier texte date de 1787 ; intitulé la Lettre du S. I. B. B. Juif de Metz à l'auteur anonyme d'un écrit intitulé : Le Cri du Citoyen contre les Juifs ${ }^{1}$, il s'agit d'une réponse d'Isaïe Berr-Bing à un opuscule hostile aux Juifs. Le second est le fameux Sermon sur la tolérance de Lazare Wogue, prononcé en $1841 .^{2}$ En soixante ans, l'appel à la tolérance a changé de destinataires : BerrBing s'adresse, à travers l'auteur du Cri, aux chrétiens, afin de défendre ses coreligionnaires des attaques dont ils sont l'objet; Wogue, quant à lui, s'adresse aux Juifs, et afin d'atténuer les dissensions au sein du judaïsme français qu'il évoque la tolérance. Cette évolution n'est pas insignifiante : elle s'ancre dans le contexte des profondes mutations que connaissent les communautés juives de France, et qui touchent tout particulièrement les Juifs de Metz.

L'apologie des Juifs messins par Isaïe Berr-Bing, lecteur de Voltaire

En 1786 paraît à Lausanne un libelle intitulé Le Cri du citoyen contre les Juifs de Metz ${ }^{3}$, dû à un capitaine d'infanterie qui reste anonyme mais qui a par la suite été identifié comme le capitaine d'infanterie Philippe-François de Latour-Foissac. ${ }^{4}$ L'auteur s'appuie sur la philosophie des Lumières, et sur la notion même de tolérance, pour dénoncer les mœurs juives. Le libelle commence en effet par ces mots :

Dans un siècle où la raison, soutenue par les dogmes sacrés d'une religion sainte, s'éclaire encore du flambeau de la Philosophie; dans un siècle où les sciences, universellement répandues, semblent avoir porté dans tous les cœurs la tolérance bienfaisante ; $(\ldots)$ un peuple sordide, couvert des épaisses ténèbres de l'ignorance et que la seule torche du fanatisme conduit encore dans les dédales obscurs de la Synagogue, lève sa tête impie et porte, sans pudeur, jusqu'aux derniers excès les plus odieuses déprédations ${ }^{5}$.

Rapidement, l'auteur s'appuie sur sur sa lecture de Voltaire, qu'il évoque sans le nommer, par périphrase, pour faire des Juifs de Metz un portrait particulièrement noir :

Indigné contre cette nation perverse, vous ne trouverez en eux, dit l'Apôtre de la tolérance, qu'un peuple ignorant et barbare, qui joint, depuis longtemps, la plus sordide avarice à la plus détestable superstition, et à la plus invincible haine pour tous les peuples qui les tolèrent, et qui les enrichissent. ${ }^{6}$

\footnotetext{
${ }^{1}$ Isaïe Berr-Bing: Lettre du S. I. B. B. Juif de Metz à l'auteur anonyme d'un écrit intitulé : Le Cri du Citoyen contre les Juifs, Metz : Colignon, 1787.

${ }^{2}$ Lazare Wogue : Sermon sur la tolérance, Metz : J. Mayer Samuel, 1841.

${ }^{3}$ Le Cri du citoyen contre les Juifs de Metz, par un capitaine d'infanterie, Lausanne, sans lieu ni date. L'ouvrage est consultable en ligne sur Gallica. Latour-Foissac est également l'auteur d'un Plaidoyer contre l'usure des Juifs des évêchés de l'Alsace et de la Lorraine, sans lieu ni date, numérisé par la Bibliothèque de l'université de Tübingen, URL : http://idb.ub.uni-tuebingen.de/diglit/FoIII918_20.

${ }^{4}$ Voir Pierre Birnbaum : "Est-il des moyens de rendre les Juifs plus utiles et plus heureux? "Le concours de l'Académie de Metz (1787), Paris : Seuil, 2017, p. 55.

${ }^{5}$ Le Cri du citoyen (note 3), p. 1.

${ }^{6} \mathrm{Ibid}$, p. 2. Latour-Foissac cite ici l'article « Juifs » du Dictionnaire philosophique, paru en 1769.
} 
La notion de tolérance, dans le discours de Latour-Foissac, s'oppose au " fanatisme » reproché aux Juifs, et ne conduit ainsi pas à une mansuétude à l'égard d'un peuple auquel Latour Foissac reproche avant tout sa pratique de l'usure.

Ce libelle vient à la connaissance du jeune Isaïe Berr-Bing, alors âgé de vingt-sept ans, qui s'est déjà illustré comme traducteur vers l'hébreu, à vingt-cinq ans, du Phaedon ou Traité sur l'immortalité de l'âme de Moïse Mendelssohn. ${ }^{7}$ C'est en homme de la Haskala ${ }^{8}$, autrement dit des Lumières juives, qu'il réagit au libelle, dont il réfute les accusations en trois points, contestant les reproches d'usure, de fanatisme et d'ignorance qui sont faites aux Juifs en reprenant et développant les points de la Bible hébraïque que le lieutenant citait en les tronquant au détriment des Juifs. Mais surtout, Berr-Bing insiste particulièrement sur le statut des Juifs en France, qui, parce qu'il ne leur permet pas d'accéder à toutes les professions, limite drastiquement et leur bonheur, et leur utilité. Je reprends ici à dessein les termes de la question mise au concours l'année même de la rédaction de la Lettre par l'Académie de Metz : «Est-il moyen de rendre les Juifs plus utiles et heureux ? ». C'est qu'en 1787, l'émancipation des Juifs n'a pas encore eu lieu : elle ne sera adoptée en France qu'en 1791 par l'Assemblée constituante, à l'initiative de l'abbé Grégoire qui avait gagné le premier prix du Concours de l'Académie de Metz. L'homme des Lumières qu'est Grégoire et le maskil, cet érudit, qu'est Berr-Bing s'accordent sur la nécessité de l'émancipation. BerrBing ne nie pas que les Juifs de Metz pratiquent le prêt d'argent (qu'il distingue de l'usure et dont il note l'utilité) et témoignent de peu d'avancement dans les lettres ; cependant, c'est leur situation politique qui les empêche de prospérer. C'est ainsi qu'il en appelle à une extension de la « tolérance » aux Juifs :

On écrivit, on disputa beaucoup en France sur la tolérance, mais on n'avait en vue que les trois sectes de religion professées dans le monde chrétien; on ne s'est pas occupé de la nôtre, les philosophes nous regardaient aussi comme trop peu importants, pour avoir pitié de notre état précaire. Je ne sais si dans ce siècle philosophique, les préjugés contre nous sont encore accrédités ; mais je sais que nous en ressentons toujours les tristes effets. Nous sommes encore constamment repoussés du sein de la patrie, on nous ferme soigneusement toutes les voies qui nous conduisent au bonheur social; on nous interdit celles dont l'homme puisse se procurer honnêtement la subsistance : nos mains sont chargées de fer, on nous fait un crime de ce que nous ne nous en servons point; et par une fatalité inconcevable, on part de là pour conclure que ne pouvant faire concourir toute notre activité au bien général, tandis que nous sommes enchaînés, nous ne le pourrions non plus quand même nos liens seraient brisés ! ${ }^{9}$

Berr-Bing constate que la tolérance professée par les philosophes des Lumières n'a encore eu d'effet que pour les protestants : il vise en cela l'Édit de tolérance de Louis XVI, qui accorde l'état-civil aux non-catholiques de France, mais dont les Juifs de Metz sont exclus explicitement. ${ }^{10}$ Le terme de tolérance, dans ce contexte, n'a pas qu'une acception morale mais a des implications politiques : l'extension nécessaire de la "tolérance » aux Juifs suppose leur émancipation, c'est-à-dire leur accès aux mêmes droits que les sujets français du roi.

\footnotetext{
${ }^{7}$ Sur la réception de Mendelssohn, voir notamment Paul H. Meyer : « Le rayonnement de Moïse Mendelssohn hors d'Allemagne », in Dix-Huitième-Siècle 13, 1981, p. 63-78.

8 Afin de défendre les Juifs de l'accusation d'ignorance, Berr-Bing mentionne d'ailleurs Mendelssohn, " généralement considéré comme le plus élégant écrivain et comme un des plus profonds métaphysiciens de son siècle ».

${ }^{9}$ Berr-Bing : Lettre (note 1), p. 27.

${ }^{10}$ Voir David Feuerwerker : L'Émancipation des Juifs en France. De l'Ancien Régime à la fin du Second Empire, Paris : Albin Michel, 1976.
} 
Berr-Bing, de ce fait, retourne les arguments voltairiens du lieutenant contre ce dernier. Il prouve, au passage, qu'il est passablement dépourvu de l'ignorance dont le lieutenant fait reproche aux Juifs de Metz, en se montrant très au fait des sources de son adversaires :

Vous avez, monsieur, des prétentions littéraires; jaloux de les faire valoir, plein de la lecture de Voltaire, vous copiez ceux des ouvrages de ce beau génie, qui sont, peut-être, les moins dignes de sa plume. C'est dans la Défense de mon Oncle, dans le Dictionnaire philosophique, dans l'Évangile du jour, et dans la Philosophie de l'Histoire que vous avez puisé vos accusations contre nous. Vous vous appropriez les injures que nous dit votre maître, vous répétez ses sarcasmes, et voilà à peu près où se bornent vos travaux. À la fin d'une longue satyre contre les Juifs, Voltaire finit par dire qu'il ne faut pourtant pas les brûler ; aussi généreux que lui, vous terminez votre diatribe en demandant la même grâce pour nous ; elle est grande, en vérité, monsieur, la grâce de passer sa vie dans l'humiliation, dans l'opprobre et dans le dénuement à peu près absolu de tous les biens de ce monde ! ${ }^{11}$

La position de Berr-Bing vis-à-vis de Voltaire est celle d'un connaisseur ayant pris ses distances. Il manifeste une réelle connaissance des œuvres de Voltaire, complétée dans une note par une mention aux théoriciens de la religion naturelle, Rousseau et "Chafisbury »" et à ce qu'il appelle le «fragile système de Spinoza ». De façon intéressante, Bing ne s'en prend pas à Voltaire pour son antijudaïsme, mais pour l'influence délétère qu'il exerce sur la moralité :

\begin{abstract}
À Dieu ne plaise, que je veuille remuer les cendres de ce grand génie que j'admire avec l'Europe entière. On doit beaucoup d'excellens ouvrages à M. de Voltaire, il a infiniment contribué à la tolérance, mais peut-être a-t-il aussi un peu contribué à beaucoup de désordres dans les mœurs. Il n'est rien de si commun dans ce siècle que ces prétendus esprits forts qui se font un plaisir d'inspirer des doutes à l'innocence, et lui enlèvent par là son unique et vraie consolation ; car assurément il n'est pas donné à tout le monde d'aimer la vertu toute nue. ${ }^{13}$
\end{abstract}

Ces propos figurent en note de bas de page afin d'étayer l'argumentation de Berr-Bing qui souhaite alors nuancer le « reproche de superstition » fait aux Juifs par le lieutenant :

Je ne sais ce que vous appelez être superstitieux; est-ce de marquer le plus inviolable attachement à une religion dans laquelle vous n'osez méconnoître le sceau de la divinité ? Est-ce d'observer très-scrupuleusement tout ce qu'elle prescrit? si c'est en cela que nous vous paroissons superstitieux, je vous avouerai volontiers que nous le sommes, que je souhaite bien sincèrement que nous le soyions toujours, en dépit des progrès de la philosophie à la mode, en dépit de son aversion pour le cérémonial, et pour tout ce qu'elle ne peut pour ainsi dire toucher au doigt. ${ }^{14}$

Il ne s'agit pas tant ici de contester Voltaire que de retourner le « reproche de superstition ». La pratique religieuse, l'observation des commandements, la célébration des fêtes étant au cœur du judaïsme, Berr-Bing ne saurait réfuter une « superstition » qui, dans une acception voltairienne, serait définie par l'attachement à la pratique plutôt qu'à l'élaboration d'une «religion naturelle» développée instinctivement par la sensibilité

\footnotetext{
${ }^{11}$ Berr-Bing (note 1), p. 5.

12 Berr-Bing écrit: "Chafisbury et Rousseau, avec leur religion naturelle épurée, ont porté, peut-être, quelqu'atteinte à celle du pays, mais ils ont raisonné, et la société n'en a pas souffert. » Sous le nom « Chafisbury », il faut sans doute reconnaître l'Evêque de Salibury selon qui « chacun sent cette Liberté [ce libre arbitre] par son expérience ", d'après le Recueil de diverses pièces sur la philosophie, la religion naturelle, \&c, compilation anonyme d'extraits de Leibniz, Newton et autres, parue à Amsterdam en 1720. Nous pouvons, de part l'approximation de cette mention chez Berr-Bing, supposer que ses connaissances en matière de théorie de la religion naturelle ne sont pas de première main.

${ }^{13}$ Ibid., p. 19-20.

${ }^{14}$ Ibid., p. 19.
} 
humaine. Berr-Bing, qui réfute méticuleusement l'accusation de fanatisme en prouvant par la citation de la Bible hébraïque que l'amour du prochain est un commandement, revendique ici une forme de «superstition » comme une garantie de morale. C'est là un argumentaire qui du reste fera florès au cours du XIX ${ }^{\mathrm{e}}$ siècle chez les Chrétiens comme chez les Juifs : l'observance religieuse est la garantie de la morale.

Il est difficile de savoir si la Lettre de Berr-Bing a été lue par son destinataire. Cela est toutefois vraisemblable si l'on considère que ce texte a circulé abondamment : il a ainsi été lu par Mirabeau, auteur en cette même année 1787 d'un essai Sur Moses Mendelssohn, sur la réforme politique des Juifs, et en particulier sur la révolution tentée en leur faveur dans la grande Bretagne. ${ }^{15} \mathrm{C}$ 'est que l'appel de Berr-Bing à l'extension de la tolérance visait bien au-delà de la communauté juive. La Lettre de Berr-Bing a ainsi eu l'effet d'une défense et illustration de la capacité des Juifs à penser et écrire en hommes des Lumières, dans le cadre idéologique français et non simplement dans celui de la Haskala allemande. L'appel à la tolérance, dont le prolongement politique résulte dans l'émancipation, résonne comme un prolongement logique de la pensée des Lumières, certes en contraste avec l'antijudaïsme voltairien, mais en revanche dans la continuité de Diderot, ${ }^{16}$ et trouve de fait des échos très concret dans les propos des penseurs chrétiens de l'époque, à commencer par l'abbé Grégoire.

\section{Le Sermon sur la tolérance de Lazare Wogue : une définition paradoxale de la tolérance?}

Les choses sont bien différentes quand Lazare Wogue prononce en 1841 son Sermon sur la tolérance, qui n'a pas pour objectif premier de convaincre un lectorat chrétien de la nécessité d'exercer la tolérance envers les Juifs. Entre 1787 et 1841, le judaïsme français a été considérablement bouleversé. D'un point de vue politique, l'émancipation en 1791 puis le décret napoléonien de 1808 ont fait des Juifs des citoyens ou sujets français, dont le culte est réglé de façon centralisée par la fondation du Consistoire israélite de France. ${ }^{17} \mathrm{La}$ fondation de l'école centrale rabbinique à Metz en 1829, transférée à Paris en 1859 sous le nom de séminaire rabbinique, encadre la formation des rabbins, dont le rôle évolue : au rabbin incarnant l'érudition et exerçant un rôle de juge au sein des communautés juives succède un rabbin incarnant une figure d'ecclésiastique et guidant une communauté, homologue juif des prêtres catholiques et des pasteurs protestants. Lazare Wogue lui-même, auteur en 1843 d'un essai intitulé Le Rabbinat français au XIX $X^{e}$ siècle ${ }^{18}$, définit les trois tâches principales du rabbin en ces termes : il est médiateur, conseiller et prédicateur. L'importance accrue de la prédication demande du rabbin une égale maîtrise de l'éloquence et de la science juive $^{19}$. Le Sermon sur la tolérance prononcé par Wogue deux ans plus tôt atteste la mise en pratique par le jeune rabbin des préceptes qu'il fixe comme idéal de sa profession, de par le succès de cette prédication, qui est imprimée, et de par l'importance des citations bibliques et talmudiques convoquées par l'orateur : il s'agit là un réel morceau d'éloquence juive.

C'est un très jeune homme qui en juillet 1841 prononce le Sermon sur la tolérance, sermon désigné par l'auteur, dans son exorde, comme un «cri que la bouche timide d'un prédicateur de vingt ans ose reproduire après le plus impétueux des prophètes d'Israël $»^{20}$.

\footnotetext{
${ }^{15}$ L'ouvrage a connu une édition moderne : Comte de Mirabeau : Sur Moses Mendelssohn, sur la réforme politique des Juifs, Paris : EDHIS ; 1968. Sur la réception de la Haskala par Mirabeau, voir Pierre Birnbaum : « Est-il des moyens de rendre les Juifs plus utiles et plus heureux? " Le concours de l'Académie de Metz (1787), p. 58 sq.

${ }^{16}$ Sur le contraste de la position de Diderot sur la question des Juifs et du judaïsme, voir notamment Elisabeth de Fontenay : Diderot ou le matérialisme enchanté, Paris : Éditions Grasset et Fasquelle, 1981.

${ }^{17}$ À ce sujet, voir Pierre Birnbaum : L'Aigle et la Synagogue : Napoléon, les Juifs et l'État, Paris : Fayard, 2007.

${ }^{18}$ Lazare Wogue : Le Rabbinat français au XIX $X^{e}$ siècle, Paris, 1843.

${ }^{19}$ Sur l'évolution de la prédication, et sur la réflexion de Wogue à ce sujet, voir l'article de Jean-Marc Chouraqui : « Le corps rabbinique en France et sa prédication : Problèmes et Desseins (1808-1905) », in Histoire, économie et société 2, 1984, p. 293-320.

${ }^{20}$ Wogue : Sermon sur la tolérance (note 2) p. 4.
} 
Lazare Wogue, futur traducteur du Pentateuque, futur enseignant très estimé au Séminaire rabbinique de Paris, est alors encore élève de l'école rabbinique de Metz. Le contexte idéologique de 1841 est tout à fait particulier : le milieu juif de Metz est en effet agité de très vives polémiques qui opposent des intellectuels réformistes aux autorités bien plus conservatrices, incarnées à Metz par Lion Mayer Lambert, Grand Rabbin depuis 1836, longtemps directeur de l'école rabbinique, jusque 1838. En 1840, Moïse-Israël Biding avait fait paraître un libelle intitulé La Vengeance d'Israë ${ }^{21}$ par lequel il répondait aux accusations de "Tsarfati le diffamateur », c'est-à-dire d'Orly Terquem. Ce dernier était un réformateur radical (il propose de célébrer le shabbat le dimanche, à titre d'exemple), du reste très isolé, qui avait déploré la misère économique et intellectuelle de la communauté de Metz. Parallèlement à ces tensions très fortes, le degré moyen de pratique religieuse et d'étude des textes juifs ne va pas en s'améliorant, ce que déplore l'éducateur Lion Mayer Lambert ${ }^{22}$.

C'est dans ce contexte difficile qu'il faut situer le sermon de Wogue, qui fait de la tolérance une vertu biblique dans un contexte interne à la communauté juive de Metz, une tolérance qui doit certes être exercée entre membres de différentes confessions, mais aussi, voire surtout au sein du Judaïsme français entre conservateurs et réformistes, afin d'éviter une scission néfaste. Sous couvert d'un propos plus général qui occupe l'essentiel du texte, le sermon se conclut par un appel à ses destinataires que sont les membres divisés de la communauté :

En effet, tous les hommes, quelles que soient leur doctrine et leur conduite, pensent et agissent à l'ordinaire, soit en conséquence de leur organisation, soit par suite des circonstances dont ils sont entourés. L'un est un fanatique, imbu, selon vous, de croyances surannées, et rebelle à toute insinuation novatrice. L'autre est un incrédule, indocile, ditesvous, au joug de notre sainte religion, ou un réformateur qui prétend la modifier d'une manière plus ou moins grave. À vous, dissidens, qui haïssez et persécutez le fanatisme ; à vous, fanatiques, qui abhorrez et maudissez l'hérésie, nous n'avons qu'une parole à vous opposer également. Faites-vous conter l'histoire de ces deux hommes. Vous verrez qu'ils obéissent l'un et l'autre à la loi de leur nature ; vous verrez que l'un et l'autre ont été dominés par des circonstances qui auraient eu sur eux un égal empire ${ }^{23}$.

C'est là la différence primordiale avec la Lettre d'Isaïe Berr-Bing : si le fait d'imprimer le Sermon en français le rend lisible au-delà des murs de la synagogue, y compris par les non-Juifs, c'est primordialement à la communauté que le discours s'adresse. Les enjeux d'une redéfinition de la tolérance sont alors différents : il s'agit pour Wogue de prouver que le mot d'ordre des Lumières n'est pas incompatible avec la religion en général, voire qu'il est préconisé par la religion juive en particulier.

L'architecture du Sermon est tout à fait conforme à ce que l'on peut attendre d'un jeune homme sur le point de sortir de l'école rabbinique. Il prend son point de départ dans la haftarah du shabbat nahamou, c'est-à-dire de la lecture imposée le jour du « shabbat de la consolation »: le verset d'Isaïe 40,3 qui figure en épigraphe est inclus dans cette haftarah. Wogue, après la captatio benevolentiae de rigueur, expose le sens de ce verset. « Un cri s'est fait entendre : Aplanissez, dans le désert, la voie du Seigneur». Les termes de « voie du Seigneur » retiennent son attention - comme son intention est manifestement d'exposer les fondements d'une tolérance compatible avec la religion juive, il s'applique à démontrer que la tolérance est constitutive de cette « voie du Seigneur », et qu'il convient de la faire advenir

\footnotetext{
${ }^{21}$ Moïse-Israël Biding : La Vengeance d'Israel. Guerre! Guerre ouverte et à outrance, pour venger les mânes de Rabbi Israel-Cohèn-Hhèzir, contre Tsarphati le diffamateur, qui l'a outragé dans le Courrier de la Moselle (Numéro du 7 mai 1839). Guerre déclarée par Moïse Israël Biding, professeur d'hébreu. Traduit de l'hébreu par L.-***, Metz, Humbert, 1840.

${ }^{22}$ Voir notamment son libelle sur l'Initiation religieuse, Metz, Imprimerie de J. Mayer Samuel 1852, ou encore la préface de la deuxième édition de son Abrégé de la grammaire hébraïque, Metz, Gerson-Lévy et Alcan, 1843.

${ }^{23}$ Wogue, Sermon sur la tolérance (note2) p. 25.
} 
dans le « désert », qu'il explique, selon une analogie absolument convenue dans le judaïsme comme dans le christianisme, comme représentatif des temps de détresse :

Or, mes frères, savez-vous bien, dites-moi, ce que c'est que le désert ? Connaissez-vous la voie du Seigneur? - Le désert, c'est l'humanité sans croyances; c'est l'humanité telle qu'elle apparaissait à Isaïe dans de sombres et désolantes visions ; c'est l'humanité de notre époque, comme le matérialisme l'a faite, comme des esprits égarés la voudraient toujours, comme le sage l'observe et la pleure ${ }^{24}$.

tolérance :

Cette «civilisation matérialiste » est pourtant celle qui lui fournit le terme de

Ce qu'il lui fallait encore, ce que demandait son cœur, ce que pressentait son génie, cette voie de Dieu qui manquait à l'humanité, parce que, pour son intelligence demi-barbare, ce grand mot, aplanir, était encore neuf et incompris... eh bien ! mes frères, je suis heureux que la langue d'une civilisation matérialiste m'en fournisse le riche équivalent, cette voie de Dieu c'était la TOLERANCE! la tolérance universelle et réciproque, généreuse et intelligente ! la tolérance, expression la plus vraie, manifestation la plus évidente de l'amour des hommes! Oui, mes frères, c'est la tolérance que proclamait le prophète, c'est elle que je viens prêcher aujourd'hui ${ }^{25}$.

Pour le jeune prédicateur, l'enjeu rhétorique est dès lors de démontrer que la tolérance est la "voie du Seigneur », qu'elle est un commandement s'imposant à tout Juif et à tout homme de bonne volonté, ce qui n'est pas sans difficulté dans la mesure où le terme est emprunté aux Lumières antireligieuses - Wogue parvient néanmoins à légitimer sa position en arguant du fait qu'il n'emprunte aux philosophes du XVIII ${ }^{e}$ siècle que le mot, et non le concept, lequel est parfaitement compatible avec la loi divine. Il s'emploie à le démontrer par de fréquentes citations de la Bible hébraïque - toujours imprimées dans les deux langues, en hébreu et en français - qu'il glose pour appuyer sa démonstration :

Enfin, mes frères ; - car, malgré la brièveté de ces citations, je crains de fatiguer votre indulgence, - il me reste à vous faire apprécier toute la réalité de la tolérance mosaïque, en appelant votre attention sur ces deux versets [lo-tišna' et-ahikha bilvakhekha] «Que la haine n'habite point dans ton cœur »; y détruire ce funeste levain qui empoisonne toutes nos affections, c'est le premier pas vers la tolérance. [hokheah tokhiah et-'amitekha velotišša 'alaiv het'] « Reprends ton prochain de ses fautes, car tu en serais puni avec lui. » Mes frères, voilà la vraie tolérance, la tolérance active, inséparable de la morale, et fondée sur cette solidarité du bien et du mal qui caractérise la société humaine. ${ }^{26}$

Mais qu'implique, concrètement, cet impératif de « tolérance »? Comment le précepte des Lumières, certes compatible avec les commandements divins, peut-il fonder des règles de comportements au sein d'une vie religieuse juive ? Avec des accents presque pascaliens, Wogue fait de la tolérance un travail d'équilibre entre deux abîmes :

Pour nous, s'il faut exposer ici notre pensée toute entière, nous le déclarons : la tolérance, vertu grande et d'un ordre supérieur, vertu éminemment philosophique, essentiellement religieuse, n'est ni indifférence, ni lâcheté, ni faiblesse ; elle est, tout au contraire, à la fois le plus grand effort de l'homme religieux contre les inspirations apparentes de sa foi, et le plus grand effort de l'homme rationnel contre les suggestions instinctives de la nature. C'est une vérité entre deux erreurs, un sentier entre deux abîmes. D’un côté, le fanatisme exclusif,

\footnotetext{
${ }^{24}$ Ibid., p. 4.

${ }^{25}$ Ibid., p. 5.

${ }^{26} \mathrm{Ibid}$, p. 17. Je transcris en caractères latins les passages bibliques cités par Wogue en hébreu consonantique.
} 
c'est-à-dire la démence d'une imagination malade ; de l'autre, l'indifférence universelle, c'est-à-dire la mort du cœur. L'homme tolérant est, avant tout, un homme sensible ${ }^{27}$.

On peinera, à la lecture du sermon, à trouver une définition de la tolérance qui échappe à ce travail d'équilibriste entre définition par la négative («ni indifférence, ni lâcheté, ni faiblesse ») ou par l'alternative (« une vérité entre deux erreurs »). En résulte l'idée d'une tolérance comme voie moyenne entre le fanatisme et l'indifférence, c'est-à-dire entre une vision radicale de la religion et un relativisme moral. Il ne s'agit pourtant pas de prêcher une pratique modérée, mais plutôt de réguler l'attitude du croyant vis-à-vis de son prochain, qu'il ne faut pas laisser dans l'erreur, mais qu'il ne faut pas non plus accabler.

Pas une fois Voltaire n'est cité par Wogue, non plus que tout autre philosophe des Lumières, contrairement à la Lettre de Berr-Bing qui montrait une connaissance des Lumières françaises et des tenants de la religion naturelle. Si Wogue ne les cite pas, c'est sans doute qu'au moment d'appuyer son Sermon sur les préceptes d'une religion trois fois millénaire, il serait malvenu de proposer un système religieux reposant uniquement sur la sensibilité humaine sans l'encadrement d'une institution religieuse. Pour autant, il trahit quelques accointances avec la pensée des Lumières, en écrivant que «l'homme tolérant est, avant tout, un homme sensible », et cette pensée de la sensibilité lui permet entre autres d'amorcer une critique de la raison, et de récuser, implicitement, les attaques voltairiennes contre les absurdités du culte juif.

Cette méfiance envers le « rationalisme exclusif » qui s'amorce dans le Sermon sur la tolérance reste une caractéristique de Wogue dans l'ensemble de ses prises de positions. Près de trente ans après la publication du Sermon, alors que Wogue est un professeur respecté du Séminaire rabbinique de Paris, il critique vigoureusement l'usage que son ancien élève Astruc fait de son nom dans l'Histoire abrégée des Juifs et de leurs croyances ${ }^{28}$. À propos de de l'enseignement qu'il a dispensé à Astruc, il écrit ainsi :

essentiellement libéral, il ne pourrait pas ne pas l'être sans mentir à son titre ; mais il y a loin, insistait-il, du libéralisme de la synagogue et des théologiens à ce rationalisme exclusif qui trône dans une partie de l'Allemagne et qu'on voudrait y importer chez nous. ${ }^{29}$

Wogue reste toute sa vie un orthodoxe, critique d'un «rationalisme excessif»: l'adhésion aux Lumières, tenable dans l'affirmation de postures morales opposées au fanatisme et dans une pensée de la sensibilité, achoppe néanmoins sur la place accordée à la raison. En ce sens, le texte de Wogue peut sembler une régression par rapport à celui de BerrBing - Wogue serait plus conservateur que Berr-Bing en ramenant la tolérance à une prescription biblique fondée sur la sensibilité humaine, sans la rationaliser outre mesure. Mais peut-être cette régression trouve-t-elle son explication dans la différence fondamentale $\mathrm{du}$ contexte dans lequel ces deux discours prennent place, car Wogue s'adresse à sa communauté, dont il essaye de préserver l'unité, et sa position est à l'aune de ce qu'il demande aux Juifs messins : une savante composition entre des forces opposées, afin de rendre possible de l'intérieur la perpétuation d'un judaïsme français.

\footnotetext{
${ }^{27}$ Ibid., p. 9.

${ }^{28}$ Élie Aristide Astruc : L'Histoire abrégée des Juifs et de leurs croyances, Paris : L. Hachette, 1869.

${ }^{29}$ Lazare Wogue : Revue israélite 10, 1870, p. 153. Sur cette polémique, voir Jean-Philippe Schreiber : «ParisBruxelles : la polémique suscitée par l'Histoire abrégée des Juifs d'Élie Aristide Astruc (1870) », in Archives Juives 40, 2007, p. 43-64. URL : https://www.cairn.info/revue-archives-juives-2007-2-page-43.htm.
} 